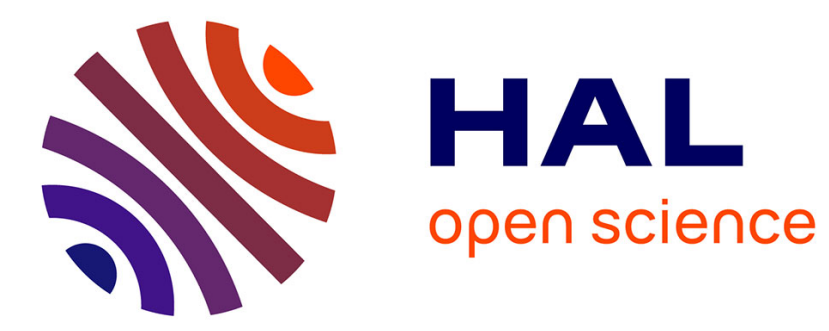

\title{
Linking the Environment, the Battery, and the Application in Energy Harvesting Wireless Sensor Networks
}

\author{
Jad Oueis, Razvan Stanica, Fabrice Valois
}

\section{To cite this version:}

Jad Oueis, Razvan Stanica, Fabrice Valois. Linking the Environment, the Battery, and the Application in Energy Harvesting Wireless Sensor Networks. AdHoc-Now 2016 - 15th International Conference on Ad Hoc Networks and Wireless, Jul 2016, Lille, France. hal-01306198

\section{HAL Id: hal-01306198 \\ https://hal.inria.fr/hal-01306198}

Submitted on 22 Apr 2016

HAL is a multi-disciplinary open access archive for the deposit and dissemination of scientific research documents, whether they are published or not. The documents may come from teaching and research institutions in France or abroad, or from public or private research centers.
L'archive ouverte pluridisciplinaire HAL, est destinée au dépôt et à la diffusion de documents scientifiques de niveau recherche, publiés ou non, émanant des établissements d'enseignement et de recherche français ou étrangers, des laboratoires publics ou privés. 


\title{
Linking the Environment, the Battery, and the Application in Energy Harvesting Wireless Sensor Networks
}

\author{
Jad Oueis, Razvan Stanica, and Fabrice Valois \\ Univ Lyon, INSA Lyon, Inria, CITI, F-69621 Villeurbanne, France \\ name.surname@insa-lyon.fr
}

\begin{abstract}
In this paper, we study photovoltaic energy harvesting in wireless sensor networks. We build a harvesting analytical model for a single node, linking three components: the environment, the battery, and the application. Given information on two of the components, limits on the third one can be determined. To test this model, we adopt several use cases with various indoor and outdoor locations, battery types, and application requirements. Results show that, for pre-defined application parameters, we are able to determine the acceptable node duty cycle given a specific battery, and vice versa. Moreover, the suitability of the deployment environment (outdoor, well lighted indoor, poorly lighted indoor) for different application characteristics and battery types is discussed.
\end{abstract}

\section{Introduction}

Wireless sensor networks (WSNs) are formed of multiple small-sized, low-cost, low-power embedded devices with sensing, computation and wireless communication capabilities [1]. The nodes self-organizing abilities, and the collaboration among them allows a wide range of applications such as military operations [2], disaster relief [3], and environmental monitoring [4], to name a few. Energy efficiency gained particular attention in WSNs design, since networks are required to run for long durations. However, node lifetime is limited by the finite capacity battery powering it. Regularly charging or replacing depleted batteries in a WSN is a complex and costly procedure, especially in large-scale networks, or in hard-to-reach deployment locations [5]. As a solution to this problem, Energy Harvesting WSNs (EH-WSNs) emerged.

EH-WSN Background. Energy harvesting consists of collecting energy from the surrounding environment and converting it to electrical energy [6]. By implementing energy harvesting, the sensor nodes become self-powered, using the renewable environmental energy as their own power source. Harvestable energy can be provided by several sources in the node ambient environment, hence the numerous energy harvesting techniques such as photovoltaic [7], piezoelectric [8], and wind energy harvesting [9]. The node energy intake depends on the output of the harvestable power source which may vary as a function of 
time. Thus, adapting power usage to the harvestable power generation pattern has been largely studied, and many harvesting-aware communication protocols were proposed [5]. In EH-WSN, the main focus shifts from energy conservation schemes to energy management, in order to optimally use harvested energy to enhance network performance [6].

Paper Contribution. In this work, we focus on photovoltaic (PV) energy harvesting. We build an analytical PV energy harvesting model for a single node in a WSN based on three components: the environment, the battery, and the application. By linking energy collection, energy management, energy consumption and the interactions between them, the operational limits of any of the three components can be specified, given information on the two other components. To test our model, we adopt several use cases covering a wide range of environments, batteries and applications. We study two application classes: outage intolerant applications, where once the node suffers from a power outage, it stops its activity permanently; outage tolerant applications, where if a power outage takes place, the node is allowed to cease its activity, recharge the battery, and then return to the network. Using our model, we are able to determine the suitability of a battery, given the node duty cycle, and vice versa. Moreover, the feasibility of an application in a particular environment can be studied. Results show that even a small power outage tolerance leads to significantly higher duty cycles.

This paper is organized as follows. Sec. 2 gives an overview on the energy harvesting model. We detail the characterization, the modeling and the adopted use cases for each of the three components: environment, battery and application in Sec. 3, Sec. 4, and Sec. 5, respectively. Results on outage intolerant applications are discussed in Sec. 6, while the outage tolerant applications are studied in Sec. 7 and Sec. 8, respectively. Finally, Sec. 9 concludes the paper, with a perspective on future work.

\section{General Objective}

Three major factors contribute to the node activity in a photovoltaic EH-WSN: the environment, the battery, and the application.

The environment, where the network is deployed, determines light intensity. Each location is distinguished by specific lighting conditions, directly affecting the energy collection process. The battery plays the part of an energy storage component and an energy provider. It impacts the node lifetime, and controls to a certain extent the energy management scheme of the node. The application sets the WSN objectives, defines requirements, such as the duty cycle, and determines the node energy consumption. Each of these components is characterized by its own set of parameters which have been largely studied in literature $[5,10-12]$. In this work, we emphasize the existing relation between these components in order to analyze the trade-offs to be considered when deploying EH-WSNs. By linking energy collection, energy management and energy consumption, that are respectively dictated by the environment, the battery and 
the application, we elaborate an energy harvesting analytical model for a single node in a WSN. Using our model, the operational limits of a component can be specified given information on the other two.

In the following, we detail each component, and the corresponding use cases adopted in the numerical analysis.

\section{The Environment Characterization}

\subsection{Energy Collection Model}

A PV panel absorbs natural or artificial light, and converts it into electrical energy that powers the node. Hence, it is the light intensity that determines the amount of energy that can be harvested by the node.

Without loss of generality, we consider in the rest of this study that time is slotted into timeslots of duration $T$ hours each. We denote by $\mathcal{T}$ the set of all timeslots $\tau$, for the whole observation period. The harvested energy during a timeslot $\tau$, denoted by $E_{h}(\tau)$, serves as input to our model. $E_{h}(\tau)$ depends on several factors such as the environment-specific light conditions, and the PV panel characteristics. We denote by $S_{P V}$ the PV panel illuminated area in $\mathrm{m}^{2}$, and by $\eta_{P V}$ the PV cell efficiency. Light intensity is modeled by the global horizontal irradiance, $I_{g h}(\tau)$, measured in $\mathrm{W} / \mathrm{m}^{2}$, representing the total amount of shortwave radiation received by the PV panel. The harvested energy by the node during timeslot $\tau$ is measured in Joules, and computed as follows:

$$
E_{h}(\tau)=I_{g h}(\tau) \cdot S_{P V} \cdot \eta_{P V} \cdot T
$$

\subsection{Use Cases}

In our analysis, we consider deployment periods of approximately one year, divided in hourly timeslots, i.e. $T=1$ hour. For the PV panel characteristics, we suppose an illuminated area $S_{P V}=10 \mathrm{~cm}^{2}$, having an efficiency $\eta_{P V}=0.25$ [13].

We consider in our analysis both outdoor and indoor deployment scenarios, covering a wide range of lighting conditions. We determine the harvested energy at each timeslot $E_{h}(\tau)$ by using real-world datasets providing measurements of the hourly $I_{g h}(\tau)$. For the outdoor scenario, we use measurements collected in Los Angeles during the year of 2014, provided by the U.S. Department of Energy [14]. The average daily irradiation is month-dependent, and varies between $39.04 \mathrm{~J} / \mathrm{cm}^{2} /$ day in December, and $109.56 \mathrm{~J} / \mathrm{cm}^{2} /$ day in June. For the indoor scenario, we consider datasets provided by Gorlatova et al. [15] presenting measurements in office buildings in New York City. We select two particular indoor locations with significantly different lighting conditions. Location A is poorly lighted, with window shading used at all times, and an average daily irradiation of $1.3 \mathrm{~J} / \mathrm{cm}^{2} /$ day. Conversely, location B is very well lighted, it has large windows with unobstructed view, and an average daily irradiation of $63 \mathrm{~J} / \mathrm{cm}^{2} /$ day. 


\section{The Battery Characterization}

\subsection{Battery Model}

In harvesting-based WSNs, nodes are usually equipped with a rechargeable battery capable of storing the harvested energy, as well as powering the node. Rechargeable batteries are mainly characterized by their capacity $E_{\max }$, and their charge/discharge efficiency $\eta_{\text {bat }}<1$ that causes energy loss when the battery is used. It should be noted that battery characteristics may vary under different operating temperatures and battery age [16]. Without loss of generality, we consider a constant discharge efficiency representing the average of the charge losses a battery may suffer from under different conditions.

In our model, we assume the presence of a power manager responsible of delivering the necessary amount of energy to the node. A node is powered, through the power manager, from the harvested energy alone, from the energy stored in the battery, or from both. The power manager has an output regulator, with an output efficiency $\eta_{\text {out }}<1$. This means that, when energy is delivered to the node, a certain amount of this energy is lost. Consequently, if we denote by $E_{c}(\tau)$ the energy consumption of a node during a timeslot $\tau$ (later detailed in Sec. 5), the power manager must deliver $E_{c}(\tau) / \eta_{\text {out }}$ Joules to the node in order to supply its demand [11].

To model the battery level variation as a function of time, we adopt the energy management model presented by Taneja et al. [11]. We extend their model by adding the battery charge/discharge efficiency $\eta_{\text {bat }}$. We denote by $E_{r}(\tau)$ the battery residual energy level at the beginning of timeslot $\tau$. At each timeslot, the node power source is determined by the power manager by comparing the amount of harvested energy $E_{h}(\tau)$, and the energy to be delivered to the node $E_{c}(\tau) / \eta_{\text {out }}$. Two cases unfold, determining the battery behavior at each timeslot:

- $E_{h}(\tau) \geq E_{c}(\tau) / \eta_{\text {out }}$ : in this case, the harvested energy is enough to solely supply the node. The remaining amount of energy, unused by the node, is stored in the battery, causing the battery to charge according to Eq. 2:

$$
E_{r}(\tau+1)=\min \left(E_{r}(\tau)+\eta_{\text {bat }} \cdot\left(E_{h}(\tau)-\frac{E_{c}(\tau)}{\eta_{\text {out }}}\right), E_{\text {max }}\right)
$$

- $E_{h}(\tau)<E_{c}(\tau) / \eta_{\text {out }}$ : in this case, the harvested energy is not enough to solely supply the node. The missing amount of energy must be provided by the battery. Consequently, two possibilities open up:

1. The amount of harvested energy, combined with the amount of the battery residual energy is enough to supply the node energy demand. The node is powered, and the battery discharges according to Eq. 3:

$$
E_{r}(\tau+1)=E_{r}(\tau)+\frac{1}{\eta_{\text {bat }}} \cdot\left(E_{h}(\tau)-\frac{E_{c}(\tau)}{\eta_{\text {out }}}\right), \text { if } E_{h}(\tau)+\eta_{\text {bat }} \cdot E_{r}(\tau) \geq \frac{E_{c}(\tau)}{\eta_{\text {out }}}
$$


2. The amount of available energy is not enough to power the node. In this case, the node is in power outage situation, and cannot be properly powered. The node must cease its activity, and we refer to it as non-operational. Let $\mathcal{T}_{\text {no }}$ be the subset of timeslots $\tau$ during which the node is non-operational:

$$
\tau \in \mathcal{T}_{\text {no }} \subseteq \mathcal{T} \Longleftrightarrow E_{h}(\tau)+\eta_{\text {bat }} \cdot E_{r}(\tau)<\frac{E_{c}(\tau)}{\eta_{\text {out }}}
$$

If power outage is tolerated, the nodes remain non-operational for a limited duration only, since the battery can still be recharged by the harvested energy. Once the available energy is sufficient again, a non-operational node resumes its activity. We consider that the tolerance for temporarily non-operational nodes depends on the specific application requirements. In this work, we study two classes of applications: outage intolerant and outage tolerant, further defined in detail in Sec. 5.

\subsection{Use Cases}

We consider in our analysis a variety of rechargeable batteries, with different characteristics. The most common rechargeable technologies are Nickel Metal Hydride (NiMH) and Lithium Ion (Li-ion). NiMH batteries suffer from low charge/discharge efficiency; however, they have a simple charging method, lowering their cost. Li-based batteries have higher charge/discharge efficiency, but a more complex charging method [5]. The set of batteries we consider in our analysis covers a wide range of characteristics, summarized in Tab. 1. Regarding the output regulator, we consider an efficiency $\eta_{\text {out }}=0.8$ [11].

\begin{tabular}{|c|c|c|c|c|c|c|}
\hline Model & Type & Volume $\left(\mathrm{cm}^{3}\right)$ & $E_{\max }(\mathrm{J})$ & Capacity $(\mathrm{mAh})$ & $\eta_{\text {bat }}$ & Notation \\
\hline AA & $\mathrm{NiMH}$ & 7.7 & 10800 & 2500 & 0.66 & AA-Ni \\
\hline AAA & $\mathrm{NiMH}$ & 3.8 & 5625 & 1250 & 0.66 & AAA-Ni \\
\hline AA & $\mathrm{Li}$ & 7.7 & 9857 & 740 & 0.99 & AA-Li \\
\hline Ultrathin_200 & $\mathrm{Li}$ & 2.7 & 2664 & 200 & 0.99 & U-Li-200 \\
\hline Ultrathin_100 & $\mathrm{Li}$ & 1.3 & 1332 & 100 & 0.99 & U-Li-100 \\
\hline Ultrathin_43 & $\mathrm{Li}$ & 0.6 & 573 & 43 & 0.99 & U-Li-43 \\
\hline Ultrathin_10 & $\mathrm{Li}$ & 0.6 & 133 & 10 & 0.99 & U-Li-10 \\
\hline
\end{tabular}

Table 1. Comparison of rechargeable batteries characteristics $[6,17]$.

\section{The Application Characterization}

\section{$5.1 \quad$ Energy Consumption Model}

The power consumption differs from one application to another, depending on the sensing activity by the sensor, the computation performed by the CPU, and 
the communication scheme of the radio transceiver. In this study, we consider that the radio transceiver is the main energy consumption source [12], disregarding other consumption sources. However, including CPU or sensing consumption in the model is straightforward. We suppose that during each timeslot $\tau$ of duration $T$, a duty cycle $D C(\tau)$ is forced on the radio transceiver. The latter will be awake, and consuming power, during each timeslot, for a time equal to $D C(\tau) \cdot T$. For the remaining time, the node is asleep, with a negligible power consumption. When awake, the transceiver switches between three states: receive, transmit, and listen. The power consumed in these states is very similar, as proven by experimental studies [12], and transceiver datasheets [18]. We consider that the power consumed by the radio while active is equal to the average of the power consumption of these three states, denoted by $P_{a v g}$. Thus, during a time period $T$, the energy $E_{c}(\tau)$ consumed by the transceiver is dictated by the duty cycle $D C(\tau)$, such as:

$$
E_{c}(\tau)=P_{a v g} \cdot D C(\tau) \cdot T
$$

\subsection{Application Classes}

The application determines the tolerance for having a temporarily non-operational node. In this study, we consider the following application classes:

Outage intolerant: For these applications, a node cannot enter the nonoperational state. The node is required to sustain its activity without suffering from a power outage, meaning that continuous operation is a requirement. We denote by $\mathcal{T}_{c o}$ the set of consecutive timeslots during which the node is operational up until the first power outage takes place. We formally define continuous operation (c.o.) as:

$$
\text { c.o. } \Longleftrightarrow E_{h}(\tau)+\eta_{\text {bat }} \cdot E_{r}(\tau)>\frac{E_{c}(\tau)}{\eta_{\text {out }}}, \quad \forall \tau \in \mathcal{T}_{\text {co }} \subseteq \mathcal{T}
$$

The node lifetime in this case is equal to the duration during which the node is continuously operational, up until the first power outage takes place. We refer to it as the continuous operation lifetime. With timeslots of duration $T$, the continuous operation lifetime is equal to $\left|\mathcal{T}_{c o}\right| \cdot T$.

Outage tolerant: For these applications, the node can be temporarily nonoperational. A node will remain non-operational as long as the amount of harvested energy combined with the battery residual energy are below the node energy requirement. Once the delivered energy is enough, the node resumes its activity. During a non-operational timeslot, the harvested energy is only used to recharge the battery:

$$
E_{r}(\tau+1)=\min \left(E_{r}(\tau)+\eta_{b a t} \cdot E_{h}(\tau), E_{\max }\right)
$$


In this work, we consider the duty cycle to be the percentage of time during which a node is active in the timeslots where the node is operational. In nonoperational timeslots, where the node is inactive, the duty cycle is null:

$$
D C(\tau)=\left\{\begin{array}{l}
D C, \quad \forall \tau \in \mathcal{T} \backslash \mathcal{T}_{\text {no }} \\
0, \quad \forall \tau \in \mathcal{T}_{\text {no }}
\end{array}\right.
$$

We limit our study to static duty cycles, such as the duty cycle is set to a fixed value $D C$ in the operational timeslots. Although dynamic duty cycles are out of the scope of this paper, expanding the framework to include adaptive duty cycles in each timeslot is straightforward.

In outage tolerant applications, it is possible that the node frequently switches between the non-operational and the operational state. We define $n_{c}$ as the consecutive number of timeslots during which the node is non-operational. Each time the node enters the non-operational state, a new instance $n_{c}(\tau)$ of the counter $n_{c}$ is created. We denote by $n_{c_{\max }}$ the maximum number of consecutive non-operational timeslots:

$$
n_{c_{\max }}=\max _{\tau \in \mathcal{T}_{\text {no }}} n_{c}(\tau)
$$

Finally, we define $r_{n o}$ as the percentage of time during which the node is non-operational out of the whole observation period $\mathcal{T}$ :

$$
r_{n o}=100 \times \frac{\left|\mathcal{T}_{\text {no }}\right|}{|\mathcal{T}|}
$$

\subsection{Use Cases}

In our analysis, we do not limit the study to specific applications, but we study different application requirements, which can be matched to a target application afterwards. Our use cases are representative of several WSN applications. We study outage intolerant applications, where continuous operation is required. We also consider several cases of outage tolerant applications with different values of $n_{c_{\max }}$ and $r_{n o}$. Regarding energy consumption, based on the CC2420 transceiver datasheet [18], we fix the energy consumption during a one hour timeslot to $P_{\text {avg }} \cdot T=180 \mathrm{~J}$.

\section{Outage Intolerant Applications}

In this section, we consider outage intolerant applications, where continuous operation is required (Eq. 6). Using our model, we are able to determine the continuous operation lifetime, i.e. the time duration before the first power shortage takes place. With timeslots of duration one hour, the continuous operation lifetime is equal to $\left|\mathcal{T}_{c o}\right|$ hours. For comparison, we use the metric $r_{c o}$, which represents the percentage of the continuous operation out of the whole observation period, such as $r_{c o}=100 \times \frac{\left|\mathcal{T}_{c o}\right|}{|\mathcal{T}|}$. 


\subsection{Outdoor}
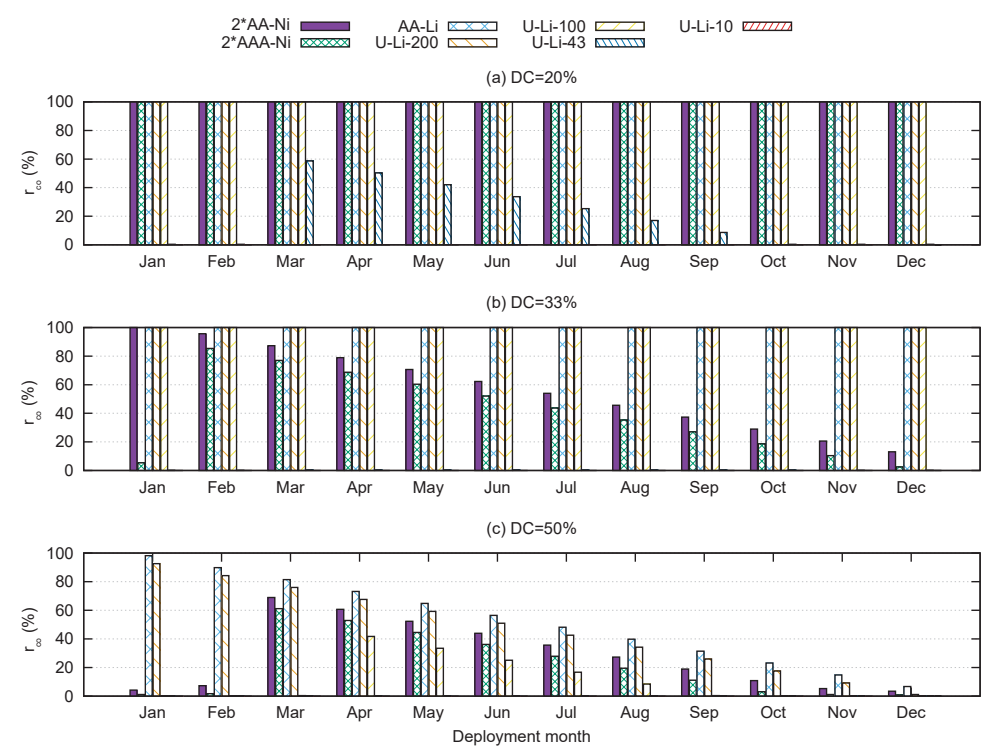

Fig. 1. Percentage of the continuous operation out of the whole observation period Outdoor.

Fig. 1 shows a comparison of $r_{c o}$ for an outdoor, one year deployment. We compare the results for the deployment starting at different months of the year. The fixed duty cycle values are such that $D C \in\{20 \%, 33 \%, 50 \%\}$. The choice of such high duty cycles is not uncommon in harvesting-based WSNs, where energy constraints are alleviated [7]. Having higher duty cycles is beneficial, since it allows lower communication delays, and higher throughput.

For $D C=20 \%$ (Fig. 1a), all the batteries are capable of guaranteeing continuous operation during the whole year, except for the smallest Li batteries. In fact, with the $10 \mathrm{mAh}$ Ultrathin battery, the node is continuously active for 2 hours only. For $D C=33 \%$ (Fig. 1b), continuous operation is achieved for a whole year for all the Li batteries, except the smallest ones, no matter the deployment month. However, for the NiMH batteries, continuous operation is only achieved for the AA-sized battery having the largest capacity, and only if the deployment starts at January. For $D C=50 \%$ (Fig. 1c), none of the batteries are capable of achieving continuous operation for a whole year, no matter the month of deployment. These results suggest that Li-based batteries generally outperform NiMH batteries. This is due to the high charging efficiency of Li batteries, as opposed to the low efficiency of NiMH batteries causing them to charge less and discharge more aggressively. On the other hand, the smallest Li batteries 
(43 and $10 \mathrm{mAh}$ ) are limited by their relatively small capacity. This suggests that much lower duty cycles are recommended when using these batteries.

A pattern concerning the effect of the deployment month on the achieved lifetime emerges. This is due to the significant difference in the amount of harvestable energy from one month to another. It is generally around November and December that the aggressive discharge will take place considering the few recharge opportunities. Thus, it is more likely that a node will enter the nonoperational state during these months.

\subsection{Indoor}

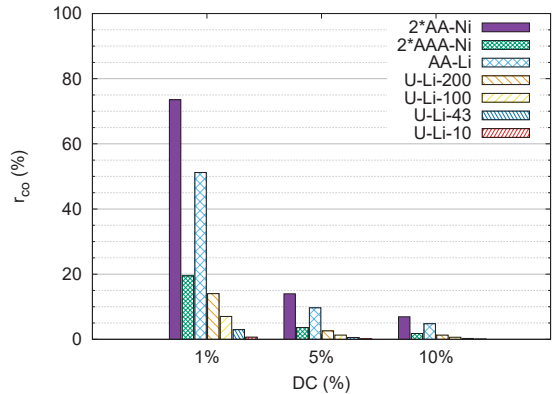

(a) Indoor Location $\mathrm{A}$

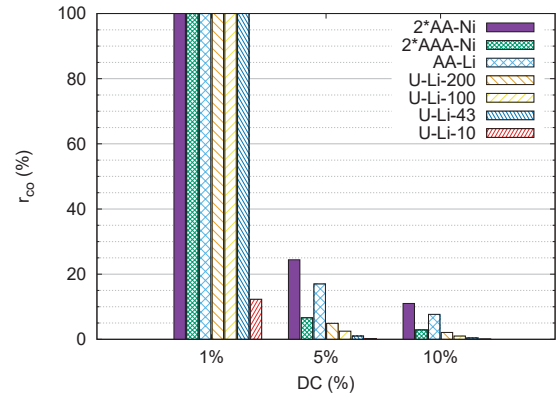

(b) Indoor Location B

Fig. 2. Percentage of the continuous operation out of the whole observation period Indoor.

Fig. 2 shows a comparison of $r_{c o}$ for the indoor locations. Since harvestable energy indoor is far less available than outdoor, smaller duty-cycles are considered: $D C \in\{1 \%, 5 \%, 10 \%\}$. Unlike the outdoor scenario, we do not have the deployment month dependency. At the well lighted location B, Fig. 2b shows that, with $D C=1 \%$, all the batteries, except the $10 \mathrm{mAh}$ Ultrathin, are capable of achieving continuous operation for the whole observation period. This means that any of these batteries can be used with a duty cycle $D C=1 \%$ or lower. Fig. 2a shows that, at the poorly lighted location A, the longest time for which the node is capable of sustaining its continuous operation is equal to $r_{c o}=73 \%$ of the observation period, for $D C=1 \%$ and two AA-sized NiMH batteries. The results show that duty cycles on the order of $D C=5 \%$ are relatively high for indoor applications, since no battery is capable of guaranteeing continuous operation for longer than $r_{c o}=24 \%$ of the observation period. Another observation in Fig. 2a is that the Ultrathin $43 \mathrm{mAh}$ and $10 \mathrm{mAh}$ batteries are only suitable for applications requiring duty cycles even lower than $D C=1 \%$. 


\section{Non-operational Time in Outage Tolerant Applications}

In this section, we consider outage tolerant applications, where continuous operation is not a requirement. The node may become non-operational for a certain duration before regaining enough energy to resume its activity. Some applications set regulations for the non-operational period, by bounding the outage tolerance parameters $n_{c_{\max }}$ (Eq. 9) and/or $r_{n o}$ (Eq. 10). Using our model, we compute $n_{c_{\max }}$ and $r_{n o}$ for a given fixed duty cycle $D C$.

\subsection{Outdoor}

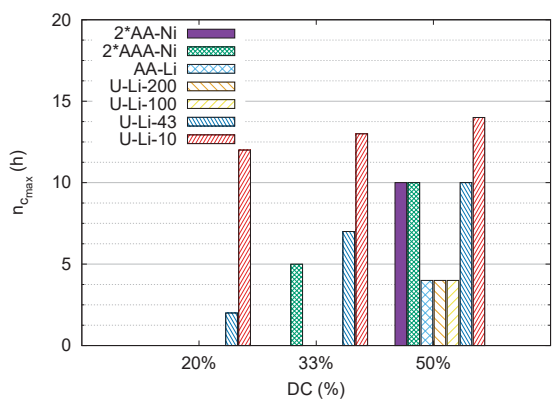

(a) Maximum consecutive non-operational hours

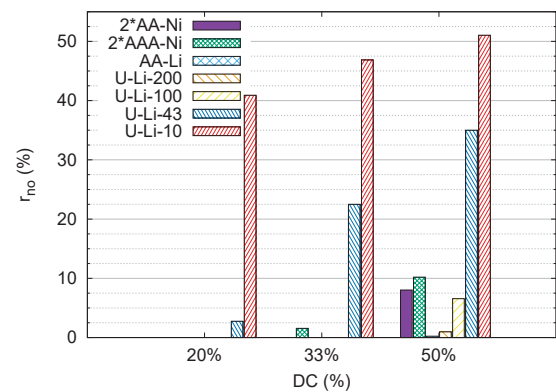

(b) Total non-operational time

Fig. 3. Outage tolerance parameters - Outdoor.

Fig. 3a and Fig. 3b show values of $n_{c_{\max }}$ and $r_{n o}$, respectively, during a one year deployment in the outdoor location, for different battery models, and for different static duty cycle values: $D C \in\{20 \%, 33 \%, 50 \%\}$. Since continuous operation is no longer a target, the month of deployment does not affect the output. Tolerance for non-operational time and the required duty cycle, set by the application, are used to determine the suitable battery. For example, a node powered by a $43 \mathrm{mAh}$ Ultrathin battery, with $D C=20 \%$, is in power outage for two consecutive hours maximum, and remains operational for a total of $97 \%$ of the whole deployment period. For applications tolerating these conditions, a node can be equipped with this type of battery. In an outage intolerant application, such batteries were not even capable of surviving one month under the same conditions.

\subsection{Indoor}

Fig. 4 shows values of $n_{c_{\max }}$ and $r_{n o}$, for indoor locations $\mathrm{A}$ and $\mathrm{B}$, for different batteries, and for different static duty cycle values: $D C \in\{1 \%, 5 \%, 10 \%\}$. In location A, Fig. 4a and Fig. 4b show that even with the relatively low $D C=1 \%$, 


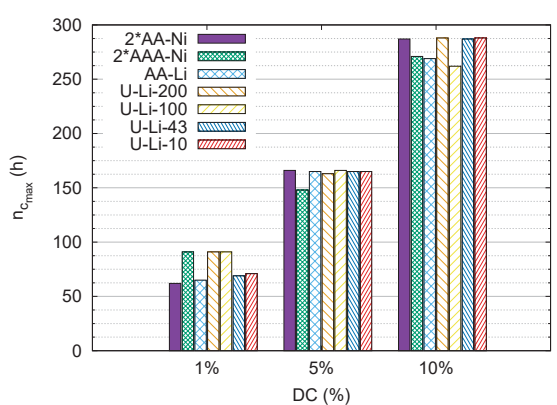

(a) Location A - Maximum consecutive non-operational hours

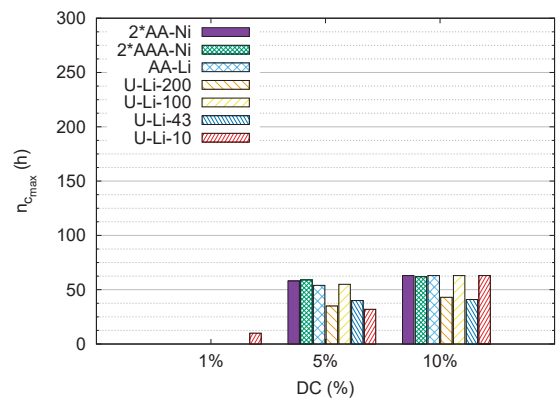

(c) Location B - Maximum consecutive non-operational hours

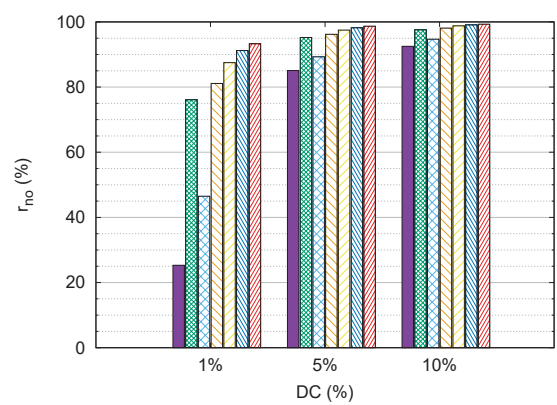

(b) Location A - Total non-operational time

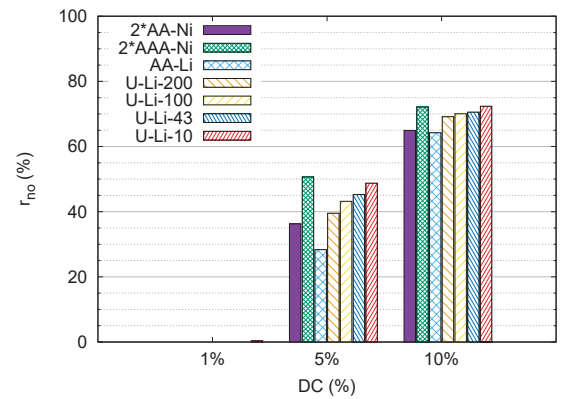

(d) Location B - Total non-operational time

Fig. 4. Outage tolerance parameters - Indoor Locations A (a,b) and B (c,d).

the tolerance for non-operational time must be high. As the duty cycle increases and the battery capacity decreases, the total non-operational time increases significantly. The values of the total non-operational time reached in location $\mathrm{A}$ (Fig. 4b), are highly impractical in real-life deployments. It is useless to have the node non-operational for $80 \%$ of the time, for example, as required by most batteries. In location B, we can see in Fig. 4d that there is no power outage for $D C=1 \%$, except with the Ultrathin $10 \mathrm{mAh}$ battery. This is in accordance with the results shown in Fig. 2b. With the Ultrathin $10 \mathrm{mAh}$ battery and $D C=1 \%$, for an outage intolerant application, the node is active for only $30 \%$ of the observation period. However, for outage tolerant applications, results show that the node is active, with $D C=1 \%$, for $96.6 \%$ of the time, while staying in the non-operational state for a maximum of 10 consecutive hours. For the higher duty cycles, total non-operational time increases significantly. 


\section{Duty Cycle Dimensioning in Outage Tolerant Applications}

We consider now the complementary problem of the previous section. Given predetermined boundaries on $n_{c_{\max }}$ and $r_{n o}$, we compute the maximum achievable $D C$ value.

\subsection{Outdoor}

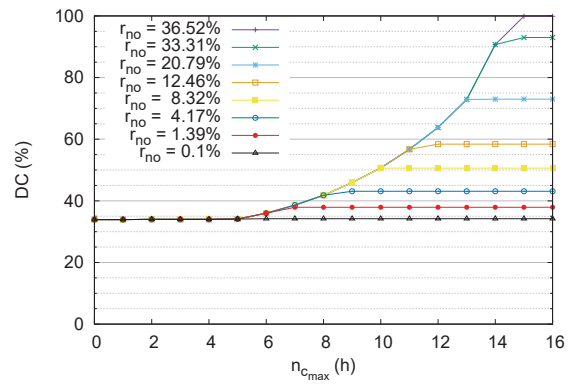

(a) Battery: $2 *$ AA-NiMH

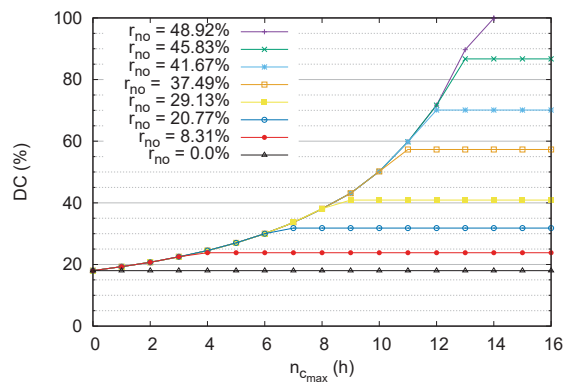

(b) Battery: U-Li-43

Fig. 5. Maximum achievable duty cycle for pre-determined conditions - Outdoor.

Fig. 5a and Fig. 5b show the maximum achievable duty cycle $D C$ in the outdoor location, with the AA-sized NiMH batteries, and the Ultrathin $43 \mathrm{mAh}$ battery, respectively. As anticipated, the more we tolerate outages, the more $D C$ increases. Since $r_{n o}$ is bounded, the maximum achievable value of $D C$ is also bounded. With the NiMH batteries, Fig. 5a shows that the maximum achievable duty cycle with no tolerance for outages is $D C=34 \%$, a relatively high value. As the tolerance for non-operational time increases, duty cycles as high as $D C=$ 93\% can be achieved, with the node being non-operational for a maximum of 15 consecutive hours, and for $33 \%$ of the observation period. However, conditions are more strict for the Ultrathin $43 \mathrm{mAh}$ battery, as shown in Fig. 5b. The maximum achievable duty cycle with no tolerance for outages is $D C=18 \%$.

\subsection{Indoor}

Fig. 6a and Fig. 6b show the maximum achievable $D C$ in indoor locations, $\mathrm{A}$ and $\mathrm{B}$, respectively, with AA-sized NiMH batteries. The difference is clear in terms of the achieved duty cycle between these two locations. In the poorly lighted location $\mathrm{A}$, the duty cycle reaches a maximum of $D C=1.3 \%$ being non-operational for $43 \%$ of the time, with 200 maximum consecutive non-operational hours. In 
the well lighted location B, the achieved duty cycle for the same non-operational conditions is $D C=5 \%$. Higher duty-cycles are achieved at the costly expense of having a large amount of time during which the node is non-operational. Some of the non-operational constraints may be impractical, meaning that applications requiring higher duty cycles as well as short outage time are difficult to accommodate to indoor environments.

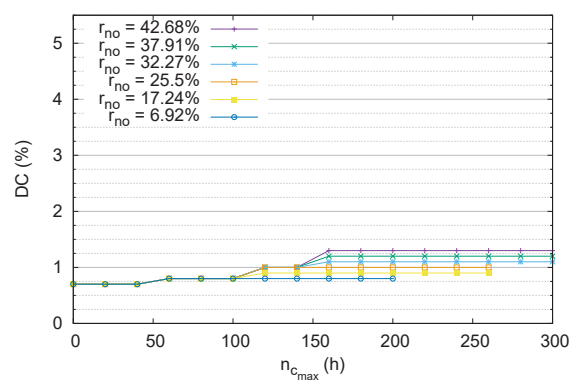

(a) Location A

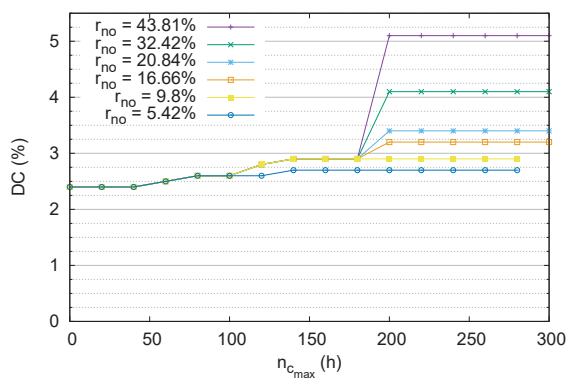

(b) Location B

Fig. 6. Maximum achievable duty cycle for pre-determined conditions with $2 *$ AANiMH batteries - Indoor.

\section{Conclusion}

In this paper, we present a PV energy harvesting model for a single node in a WSN. We build our model around three major components: the environment, the battery, and the application. Based on our model, given two of the three components, we can characterize the third one. We define several use cases using real-world datasets of light intensity, in both indoor and outdoor scenarios, a wide range of rechargeable batteries, and various application parameters. We show that, given particular application parameters, we are able to determine which batteries are suitable, given the node duty cycle, and vice versa. Furthermore, the feasibility of an application in a specific environment, or for a specific battery, can be studied. Results show that tolerating short power outage periods allows extending the node lifetime, with higher duty cycle values.

For future work, it is of utmost importance to further expand the model from a single wireless node to the whole network. Collaboration between the nodes, and relaying functionalities may influence the node behavior. Moreover, the outage tolerant applications raise new challenges on how to deal with the unexpected "disappearance" of a node when non-operational, followed by its "reappearance" in the network. 


\section{References}

1. J. Yick, B. Mukherjee, D. Ghosal, "Wireless Sensor Network Survey", Computer Networks, 52(12), 2292-2330, 2008.

2. S. H. Lee, S. Lee, H. Song, H. S. Lee, "Wireless Sensor Network Design for Tactical Military Applications: Remote Large-scale Environments", Proc. IEEE Milcom 2009, Boston, MA, USA, 2009.

3. D. Chen, Z. Liu, L. Wang, M. Dou, J. Chen, H. Li, "Natural Disaster Monitoring with Wireless Sensor Networks: A Case Study of Data-intensive Applications Upon Low-cost Scalable Systems", Mobile Networks and Applications, 18(5), 651-663, 2013.

4. L. Oliveira, J. Rodrigues, "Wireless Sensor Networks: A Survey On Environmental Monitoring", Journal of Communications, 6(2), 143-151, 2011.

5. S. Basagni, M. Y. Naderi, C. Petrioli, D. Spenza, "Wireless Sensor Networks with Energy Harvesting", Mobile Ad Hoc Networking: The Cutting Edge Directions, pp. 701-736, 2nd ed., Wiley, 2013.

6. S. Sudevalayam, P. Kulkarni, "Energy Harvesting Sensor Nodes: Survey and Implications", IEEE Communications Surveys \& Tutorials, 13(3), 443-461, 2011.

7. V. Raghunathan, A. Kansal, J. Hsu, J. Friedman, M. Srivastava, "Design Considerations for Solar Energy Harvesting Wireless Embedded Systems", Proc. ACM/IEEE IPSN 2005, Los Angeles, CA, USA, 2005.

8. K. Vijayaraghavan, R. Rajamani, "Active Control Based Energy Harvesting for Battery-less Wireless Traffic Sensors: Theory and Experiments", Proc. IEEE ACC 2008, Seattle, WA, USA, 2008.

9. A. Cammarano, D. Spenza, C. Petrioli, "Energy-harvesting WSNs for structural Health Monitoring of Underground Train Tunnels", Proc. IEEE Infocom Student Workshop, Turin, Italy, 2013.

10. M. Gorlatova, A. Wallwater, G. Zussman, "Networking Low-Power Energy Harvesting Devices: Measurements and Algorithms", IEEE Transactions on Mobile Computing, 12(9), 1853-1865, 2013.

11. J. Taneja, J. Jeong, D. Culler, "Design, Modeling, and Capacity Planning for Micro-solar Power Sensor Networks", Proc. ACM/IEEE IPSN 2008, St Louis, MO, USA, 2008.

12. A. Prayati, C. Antonopoulos, T. Stoyanova, C. Koulamas, G. Papadopoulos, "A Modeling Approach on the TelosB WSN Platform Power Consumption", Journal of Systems and Software, 83(8), 1355-1363, 2010.

13. M. Green, K. Emery, Y. Hishikawa, W. Warta, and E. Dunlop, "Solar Cell Efficiency Tables (v. 45)", Progress in Photovoltaics: Research and Applications, 23(1):1, 2015.

14. A. Andreas, S. Wilcox, "Solar Resource \& Meteorological Assessment Project (SOLRMAP)', Rotating Shadowband Radiometer (RSR), NREL Report No. DA5500-56502, Los Angeles, CA, USA, 2012

15. M. Gorlatova, M. Zapas, E. Xu, M. Bahlke, I. Kymissis, G. Zussman, CRAWDAD Dataset Columbia/Enhants, http://crawdad.org/columbia/enhants/2011040\%, 2011, Accessed: Apr. 2016.

16. I. Buchmann, "Batteries in a Portable World: A Handbook on Rechargeable Batteries for Non-engineers", Cadex Electronics, 2nd ed., 2001.

17. Ultrathin Rechargeable Lithium Polymer Batteries from Powerstream, http://www.powerstream.com/thin-lithium-ion.htm, Accessed: Apr. 2016.

18. Texas Instruments, "Chipcon CC2420 Datasheet", http://www.ti.com/lit/ds/symlink/cc2420.pdf, Accessed: Apr. 2016. 